\title{
Efeitos da música eletrônica nos sistemas neuromuscular, cardiovascular e parâmetros psicofisiológicos durante teste incremental exaustivo
}

\author{
Electronic music effects on neuromuscular and cardiovascular systems \\ and psychophysiological parameters during exhaustive incremental test
}

\author{
B.P.C. Smirmaul, J.L. Dantas, E.B. Fontes, A.C. Moraes
}

RESUMO

O objetivo do presente estudo foi analisar o efeito da música sobre respostas fisiológicas e psicofisiológicas, assim como sobre a potência máxima alcançada durante um teste incremental. Uma amostra de 10 indivíduos saudáveis $(20.8 \pm 1.4$ anos, $77.0 \pm 12.0 \mathrm{~kg}, 179.2 \pm 6.3 \mathrm{~cm})$ participou deste estudo. Foram coletadas a atividade eletromiográfica (músculos Reto Femoral - RF e Vasto Lateral - VL), a frequência cardíaca (FC), a percepção subjetiva de esforço (PSE), a percepção subjetiva de tempo (PST) e a potência máxima alcançada (PMax) durante as situações com música (CM) e sem música (SM). Os indivíduos completaram quatro testes incrementais máximos (TIMax) do tipo rampa em um ciclossimulador com uma carga inicial de $100 \mathrm{~W}$ e aumentos de $10 \mathrm{~W} \cdot \mathrm{min}^{-1}$. As médias dos valores de PMax entre as situações SM $(260.5 \pm 27.7 \mathrm{~W})$ e CM $(263.2 \pm 17.2 \mathrm{~W})$ não apresentaram diferença significativa. A comparação entre as taxas de aumento dos valores expressos em root-meansquare (RMS) e frequência mediana (FM) para os dois músculos (RF e VL) também não apresentaram diferença significativa, assim como a FC, PSE e PST. Conclui-se que a música eletrônica durante um teste incremental até a exaustão não surtiu efeito sobre as variáveis analisadas para o grupo investigado.

Palavras-chave: eletromiografia, música, ciclismo, frequência cardíaca, percepção de esforço

ABSTRACT

The aim of this study was to analyze the music effects on physiological and psychophysiological responses, as well as on the maximum power output attained during an incremental test. A sample of 10 healthy individuals $(20.8 \pm 1.4$ years, $77.0 \pm 12.0 \mathrm{~kg}, 179.2 \pm 6.3 \mathrm{~cm})$ participated in this study. It was recorded the electromyographic activity (muscles Rectus Femoris - RF and Vastus Lateralis - VL), heart rate (HR), rating of perceived exertion (RPE), ratings of perceived time (RPT) and the maximum power output attained (PMax) during music (WM) and without music (WTM) conditions. The individuals completed four maximal incremental tests (MIT) ramp-like on a cycle simulator with initial load of $100 \mathrm{~W}$ and increments of $10 \mathrm{~W} \cdot \mathrm{min}^{-1}$. The mean values of PMax between conditions WTM $(260.5 \pm 27.7 \mathrm{~W})$ and WM $(263.2 \pm 17.2 \mathrm{~W})$ were not statistically different. The comparison between the rates of increase of the values expressed in root-mean-square (RMS) and median frequency (MF) for both muscles (RF and VL) also showed no statistical difference, as well as HR, RPE and RPT. It is concluded that the use of the electronic music during an incremental test to exhaustion showed no effect on the analyzed variables for the investigated group.

Keywords: electromyography, music, cycling, heart rate, rating of perceived exertion

Submetido: 25.07.2010 | Aceite: 12.09.2010

Agradecimentos. Os autores agradecem o apoio financeiro proporcionado pelo Programa Institucional de Bolsas de Iniciação Científica - PIBIC/CNPq - PRP. FAPESP Processo 04-12589-0 e 05-00151-2

Bruno de Paula Caraça Smirmaul, Eduardo Bodnariuc Fontes e Antonio Carlos de Moraes. Universidade Estadual de Campinas (UNICAMP), Campinas - SP, Brasil.

José Luiz Dantas. Universidade Estadual de Londrina (UEL), Londrina - PR, Brasil.

Endereço para correspondência: Bruno de Paula Caraça Smirmaul, Avenida Érico Veríssimo, nº 701, Cidade

Universitária Zeferino Vaz, Barão Geraldo, CEP: 13083-851 Campinas/ SP, Brasil.

E-mail: brunosmirmaul@gmail.com 
A psicofisiologia é um ramo da fisiologia que estuda a inter-relação entre os fenômenos físicos e mentais (Random House, 2005). Segundo Cacioppo, Tassinary e Berntson (2000), a psicofisiologia pode ser descrita como uma extensão natural da anatomia e da fisiologia objetivando entender cientificamente o comportamento e os processos humanos, inter-relacionando o corpo humano com $\mathrm{o}$ ambiente. Os conceitos de psicofisiologia têm sido cada vez mais utilizados por pesquisadores no ramo do esporte e do exercício, a fim de elucidar questões referentes aos processos do sistema nervoso central e suas influências na atividade física (Kayser, 2003; Noakes, St Clair Gibson, \& Lambert, 2005).

Dentre os vários aspectos da psicofisiologia, uma relação que tem sido abordada é a influência da música no organismo humano. Essas investigações variam desde estudos sobre o impacto na dor (Cepeda, Carr, Lau, \& Alvarez, 2006; Klassen, Liang, Tjosvold, Klassen, \& Hartling, 2008), na recuperação de lesões (Sarkamo et al., 2008), na cognição (Mammarella, Fairfield, \& Cornoldi, 2007), até o impacto da música na atividade física (Etzel, Johnsen, Dickerson, Tranel, \& Adolphs, 2006; Karageorghis, Jones, \& Stuart, 2008; Khalfa, Roy, Rainville, Dalla Bella, \& Peretz, 2008). Simpson e Karageorghis (2006) mencionam que a música é um recurso de motivação e inspiração muito valioso no campo do esporte e do exercício. Considerando ainda que a fadiga possa ser vista como uma percepção sensorial, muito além de apenas um evento físico (St Clair Gibson \& Noakes, 2004), a música poderia, de alguma forma, alterar seu processo, retardando o ritmo de sua instalação.

A utilização de músicas do tipo "eletrônica" pela maioria das academias parte de encontro com os achados de Karageorghis, Jones e Low (2006), que constataram uma significante preferência pelas músicas rápidas (140 bpm), comparado às médias (120 bpm) e lentas (80 $\mathrm{bpm}$ ). Diversos estudos revelaram mudanças psicofisiológicas no organismo perante a música (Boutcher \& Trenske, 1990; Copeland \& Franks, 1991; Karageorghis \& Terry, 1997; Lee, 1989; Lucaccini \& Kreit, 1972), sendo a Teoria da Atenção Restrita (Hernandez-Peon, Brust-Carmona, Penaloza-Rojas, \& Bach-YRita, 1961) uma das mais citadas para explicar essas mudanças. Segundo essa teoria, a quantidade de estímulos que podem ser processados em um único momento pelo sistema nervoso é limitada. Sendo assim, uma música estimulante e/ou agradável poderia inibir as sensações "desagradáveis" associadas à fadiga (Tenenbaum et al., 2004), diminuindo a atenção do indivíduo a essas sensações e consequentemente aumentando a motivação para a tarefa e protelando o ponto de exaustão.

A música se tornou elemento indispensável para a maioria dos praticantes de atividade física, principalmente dentro de academias de ginástica. $\mathrm{O}$ aumento da utilização dos aparelhos musicais portáteis permite que muitos usufruam desse instrumento durante a prática de exercícios físicos. Apesar dessa massiva utilização e aumento do interesse de pesquisadores nessa área, os efeitos da música durante o exercício têm sido estudados principalmente analisando-se a percepção de esforço (campo psicológico) e as respostas cardíacas (campo fisiológico) (Karageorghis et al., 2006; Karageorghis \& Terry, 1997; Yamashita, Iwai, Akimoto, Sugawara, \& Kono, 2006).

No entanto, dentro do nosso conhecimento, pouco se sabe sobre as respostas do sistema neuromuscular nessa situação. Diante da perspectiva supracitada sobre o efeito ergogênico da música, relacioná-la com parâmetros neuromusculares pode ampliar e trazer novos achados a respeito dos efeitos da música no exercício, esclarecendo melhor como se dá essa influência.

Assim, o objetivo do presente estudo foi analisar o efeito ergogênico da música sobre as respostas fisiológicas (sinal eletromiográfico EMG e frequência cardíaca), psicofisiológicas (percepção subjetiva de esforço e percepção subjetiva de tempo) e desempenho (potência 
máxima) alcançado durante a realização de um teste incremental exaustivo.

\section{MÉTODO}

\section{Amostra}

Participaram do estudo 10 voluntários saudáveis $(20.8 \pm 1.4$ anos, $77.0 \pm 12.0 \mathrm{~kg}$, $179.2 \pm 6.3 \mathrm{~cm}$ ), sem histórico de lesões musculares nos membros inferiores nos seis meses anteriores ao experimento. Os voluntários eram fisicamente ativos (2-4 sessões semanais de atividades físicas recreacionais), porém, não treinados. Todos foram orientados a se alimentarem e se hidratarem normalmente no período anterior aos testes, não ingerindo qualquer tipo de bebida que pudesse afetar o rendimento (bebidas energéticas, alcoólicas, cafeinadas, etc.) e também a não praticarem exercícios físicos vigorosos nas 24 horas precedentes aos testes.

Todos os voluntários assinaram o termo de consentimento livre e esclarecido, após serem devidamente informados sobre os detalhes e procedimentos do presente estudo, o qual foi previamente aprovado pelo Comitê de Ética e Pesquisa (CEP) da Faculdade de Ciências Médicas (FCM) da Universidade Estadual de Campinas (UNICAMP) sob protocolo número $626 / 2008$, de acordo com as normas da Resolução 196/96 do Conselho Nacional de Saúde sobre pesquisa envolvendo seres humanos.

\section{Instrumentos e Procedimentos}

\section{Delineamento Experimental}

As coletas do presente estudo foram realizadas durante um período de seis semanas. Após um aquecimento de um minuto no ciclossimulador a $150 \mathrm{~W}$, os indivíduos realizaram, em dias diferentes, quatro testes incrementais máximos (TIMax) do tipo rampa em um ciclossimulador modelo CompuTrainer DYNAFIT $^{\mathrm{TM}}$ (RacerMate ${ }^{\circledR}$, Seattle, WA, USA) com uma carga inicial de $100 \mathrm{~W}$ e aumentos de $10 \mathrm{~W} \cdot \mathrm{min}^{-1}$, até a exaustão voluntária ou incapacidade de manter a cadência estipulada de 60 rotações por minuto (rpm) por mais de cinco segundos.

Os dois primeiros TIMax tiveram como objetivo a familiarização com os procedimentos, sendo que um foi realizado com música (CM) e o outro sem música (SM). $\mathrm{O}$ terceiro e o quarto TIMax também consistiram das mesmas duas situações (CM e SM). Tanto a ordem dos testes de familiarização, como dos dois últimos foi contrabalanceada.

Os indivíduos foram orientados a se concentrarem para pedalar o maior tempo possível, uma vez que não houve estímulo verbal durante nenhuma das condições de teste, visando evitar outro fator influenciador na motivação dos voluntários, além da música. Ajustes de altura no ciclossimulador foram reproduzidos durante todos os testes, evitando assim interferência nos resultados (Ashe et al., 2003).

As músicas utilizadas foram do gênero "eletrônico", comumente usadas em academias, e utilizou-se o software Audacity ${ }^{\circledR}$ 1.3.3 (Microsoft Windows) para garantir uma batida superior a $130 \mathrm{bpm}$. Todos os testes da situação CM foram realizados com a mesma sequência de músicas e intensidade de som. Foi utilizado um fone de ouvido Headset Profissional (Bright, USA). O intervalo entre cada teste para um mesmo indivíduo foi de, no mínimo, 48 horas.

Durante todos os testes foram coletados: a atividade eletromiográfica (ver Coleta e Processamento das Variáveis), a frequência cardíaca (FC), a percepção subjetiva de esforço (PSE), a percepção subjetiva de tempo (PST) e a potência máxima alcançada (PMax).

A FC foi aferida com um cardiofrequencímetro da marca Polar, modelo S810I (POLAR ${ }^{\circledR}$, Finlândia).

A PSE foi aferida a cada dois minutos de teste através da escala de 15 pontos (6-20) de Borg (Borg, 1982), que foi fixada em frente aos voluntários, objetivando a fácil visualização pelos mesmos. Para todos os experimentos os voluntários foram instruídos a responder a 
questão: "O quão intenso está a tarefa neste momento?” (Borg, 1970; Garcin, Fleury, MilleHamard, \& Billat, 2005). Os valores para percepção extremamente fácil (7) e o valor extremamente difícil (19) serviram de âncora para a instrução dos voluntários.

Já a PST foi aferida através de uma pergunta ("Quanto tempo acha que passou desde o início do teste?"). Vale ressaltar que os indivíduos não possuíam acesso a nenhuma informação (tempo decorrido e FC).

\section{Coleta e Processamento das Variáveis}

Foram analisados os sinais eletromiográficos dos músculos Reto Femoral (RF) e Vasto Lateral (VL), dois importantes músculos solicitados durante o ciclismo (Lucia, Sanchez, Carvajal, \& Chicharro, 1999). Para a coleta dos sinais eletromiográficos foi utilizado um eletromiógrafo da marca BIOPAC (BIOPAC System, Inc., Santa Barbara, CA, USA), modelo MP150, contendo 16 canais. A frequência de aquisição dos sinais eletromiográficos foi estabelecida em $2000 \mathrm{~Hz}$ e o filtro passa-banda aplicado foi de 20-500 Hz. Os limites de entrada dos sinais foram estabelecidos em \pm 5 $\mathrm{mV}$. Para a captação e processamento dos sinais foi utilizado o software AcqKnowledge 3.8.1 (BIOPAC System, Inc., Santa Barbara, CA, USA). Inicialmente, foi realizada assepsia dos locais utilizando álcool, seguido de curetagem para reduzir a impedância da pele. Para a coleta dos sinais eletromiográficos foram utilizados eletrodos ativos (BIOPAC System, Inc., Santa Barbara, CA, USA) modelo TSD 150B (CMRR - relação de rejeição do modo comum $>95 \mathrm{~dB}$ ), que foram dispostos nos músculos analisados conforme posicionamento de referência descrito por Hermens, Freriks, Disselhorst-Klug e Rau (2000).

Para o músculo RF, o eletrodo foi posicionado no ponto mediano da distância entre a crista ilíaca ântero-superior e a borda superior da patela, e para o músculo VL, foi utilizado o ponto localizado a dois terços da distância entre a crista ilíaca ântero-superior e a borda lateral da patela. Os eletrodos foram colocados nos músculos do membro direito do voluntário, sendo a distância intereletrodos fixada em dois centímetros (centro a centro). Foi utilizada fita adesiva (Transpore, $3 \mathrm{M}$ do Brasil, Inc.) para a fixação dos eletrodos sobre a pele. O eletrodo de referência (terra) foi posicionado na protuberância óssea do acrômio do lado direito do voluntário.

Após coletados, os dados foram filtrados e expressos em root mean square (RMS) e frequência mediana (FM). Cada ponto foi calculado a partir de uma média a cada período de dois minutos. Para o cálculo da FC foram utilizados os 15 segundos precedentes e posteriores a cada período de dois minutos de teste, totalizando 30 segundos em cada amostragem (ex: para o primeiro ponto, utilizava-se do tempo $1: 45$ ao $2: 15$, para o segundo ponto, do tempo 3:45 ao 4:15, e assim por diante). Anotaram-se os valores da taxa de aumento (SLOPE) e coeficiente de explicação $\left(R^{2}\right)$ das variáveis EMG, obtidos por meio de regressão linear dos pontos em relação ao tempo, adquirindo assim, uma informação global do comportamento de cada variável ao longo de todo o teste, sendo o mesmo feito para a análise da FC.

O mesmo procedimento também foi realizado para as outras variáveis (PSE e PST), porém com os valores obtidos a cada dois minutos para PSE, enquanto para a PST os períodos eram de três em três minutos. Para mascarar o efeito da temporização da sequência de perguntas nas respostas de PST, a PSE era solicitada também em momentos variados, fora do período de dois minutos, porém, essas não eram anotadas.

\section{Análise Estatística}

Primeiramente os dados foram submetidos à estatística descritiva. Posteriormente foi aplicado o teste de Shapiro-Wilk para testar a normalidade dos dados.

Para comparação da PMax entre as situações CM e SM, assim como para comparação entre os SLOPES das variáveis 
RMS, FM, FC, PSE e PST, foi utilizado o teste $\mathrm{t}$ de Student para amostras dependentes.

Os dados de desempenho (PMax) foram expressos em média e desvio padrão $(M \pm D P)$, enquanto os dados fisiológicos e psicofisiológicos foram expressos em média, erro padrão (EP), valor mínimo e máximo. O coeficiente de determinação $\left(R^{2}\right)$ da regressão para determinação dos coeficientes de inclinação (SLOPE) das variáveis analisadas também foi utilizado para demonstrar a explicação compartilhada da variação dos índices fisiológicos em relação ao tempo em teste, permitindo verificar se a música influenciaria a dispersão da relação entre as variáveis. Os dados de $R^{2}$ das variáveis RMS, FM, FC, PSE foram contrastados por meio de teste $\mathrm{t}$ de Student para amostras dependentes, e para a variável PST foi utilizado o teste nãoparamétrico de Wilcoxon.

O nível de significância adotado foi de $p<$ .05 .

\section{RESULTADOS}

As médias dos valores de PMax entre as situações não apresentaram diferença estaticamente significante $(p>.05)$ (Figura 1$)$.

A comparação entre os SLOPES das

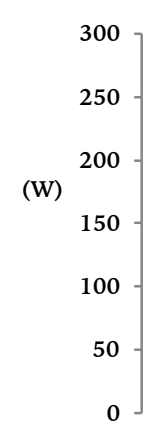

Potência Máxima Alcançada (PMax)

Figura 1. Valores da potência máxima alcançada (PMax) durante os TIMax nas condições sem música (SM) e com música (CM)

variáveis RMS e FM para os dois músculos (RF e VL) também não apresentaram diferença significativa, assim como a FC, PSE e PST ( $p>$ .05) (ver Tabela 1$)$. De todos os voluntários ( $n$ $=10$ ), apenas o sinal do músculo RF de um sujeito teve que ser retirada, devido à má qualidade do sinal. Não houve diferença significativa para nenhuma comparação de SLOPES entre as situações SM e CM $(p>.05)$.

\section{DISCUSSÃo}

Há divergência na literatura quanto ao efeito ergogênico da música em exercícios de moderada a alta intensidade (Nakamura,

Tabela 1

Taxa de aumento (SLOPE) expressos em média, erro-padrão (EP), mínimo, máximo e $R^{2}$ das variáveis $R M S\left(m V .2 m^{-1}{ }^{-1}\right) e$

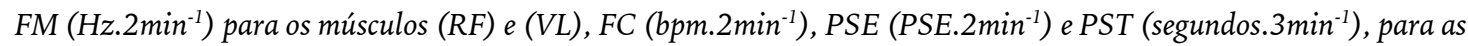
condições com música (CM) e sem música (SM)

\begin{tabular}{lccccccc}
\hline & RMS - RF & RMS - VL & FM - RF & FM - VL & FC & PSE & PST \\
\hline SLOPE SM & .026 & .015 & -.002 & -.004 & 9.4 & 1.9 & 193.2 \\
EP & .007 & .002 & .004 & .002 & .5 & .1 & 6.9 \\
Mínimo & .003 & .006 & -.017 & -.011 & 7.0 & 1.5 & 165 \\
Máximo & .078 & .031 & .020 & .004 & 13.4 & 2.4 & 240 \\
$R^{2}$ & .581 & .790 & .412 & .384 & .99 & .98 & .99 \\
\hline SLOPE CM & .022 & .013 & -.006 & .002 & 9.6 & 1.8 & 206.4 \\
EP & .004 & .002 & .003 & .005 & .4 & .1 & 9.0 \\
Mínimo & .008 & .000 & -.021 & -.008 & 7.5 & 1.4 & 167 \\
Máximo & .050 & .029 & .014 & .044 & 11.5 & 2.2 & 240 \\
$R^{2}$ & .758 & .677 & .467 & .410 & .99 & .98 & .99 \\
\hline
\end{tabular}


Pereira, Papini, Nakamura, \& Kokubun, 2010), acarretando em um prolongamento do exercício, o que em nosso estudo seria representado por uma PMax superior na condição CM. Com esse prolongamento, poderia haver mudanças nos parâmetros relacionados com a exaustão e tolerância ao exercício (EMG, FC, PSE e PST). Porém, os resultados do presente estudo demonstraram que isso não ocorreu. Os resultados indicam que a música eletrônica não alterou o desempenho entre as duas situações estudadas.

Segundo a Teoria da Atenção Restrita de Hernandez-Peon et al. (1961), o cérebro possui uma quantidade limitada de informações que podem ser processadas ao mesmo tempo e, a música, acabaria por inibir sensações "desagradáveis" resultante do exercício físico, atenuando a sensação de esforço do indivíduo. Essa teoria tem sido utilizada para explicar os efeitos da música durante o exercício físico (Karageorghis \& Terry, 1997; Szmedra \& Bacharach, 1998). Nossa hipótese era de que com uma atenuação da sensação de esforço, um prolongamento do exercício poderia ocorrer, causando assim, mudanças não apenas sensoriais, mas também metabólicas. Ao contrário de Szmedra e Bacharach (1998) que encontraram diminuição significativa da PSE durante um protocolo submáximo a $70 \%$ do $\mathrm{VO}_{2}$ máx com a utilização de música, o presente estudo não demonstrou efeito da música nesta variável. Boutcher e Trenske (1990) também encontraram redução da PSE com utilização de música, porém, apenas para a intensidade mais leve. Durante as intensidades moderada e pesada, a música não surtiu efeito sobre a PSE. O fato do presente estudo não verificar nenhuma alteração nas respostas da PSE pode dar-se pelo fato de que, apesar dos voluntários relatarem se sentirem mais motivados para pedalar com música, nosso protocolo consistiu em um teste incremental até a exaustão, em que provavelmente o constante aumento da carga impossibilitou que a música exercesse algum tipo de efeito ergogênico.
Quanto à FC, Szmedra e Bacharach (1998) também encontraram significante diminuição em determinados pontos durante o protocolo aplicado com música. Já nosso estudo não encontrou diferença entre os SLOPES de FC. Uma possibilidade para o ocorrido, é que utilizamos música do gênero "eletrônica", com batidas rápidas, enquanto Szmedra e Bacharach (1998) utilizaram música clássica para seu protocolo. Essa diferença de gênero musical pode ter forte influência nas respostas geradas pela música, sendo um fator muito importante, como frisado no artigo de revisão de Karageorghis e Terry (1997). Outra variável analisada nesse estudo foi a PST, a fim de explorar se a música poderia causar algum efeito na percepção de tempo dos indivíduos. Caso houvesse alguma mudança nessas respostas, poderíamos ver na música uma possibilidade de "enganar" o cérebro, fazendo o sujeito achar que ficou menos tempo na situação com música, quando na verdade ficou o mesmo tempo. Entretanto, os resultados mostraram que PST não foi diferente para as duas situações. Essa variável pode servir, em estudos futuros, como mais uma fonte de informação para avaliação subjetiva de percepção durante diferentes protocolos.

A inovação deste estudo se deu na análise do comportamento do sistema neuromuscular durante o exercício físico com e sem utilização da música. O efeito ergogênico da música sobre o comportamento da RMS ou da FM não foi confirmada, assim como sobre o desempenho. Apesar disso, os resultados deste estudo permitem um passo importante na compreensão dos efeitos da interação entre o comportamento do sistema neuromuscular e a música durante exercício. Nossos dados, comparado com os estudos anteriormente citados, permitem supor que exista uma dependência da tarefa para que ocorra o efeito da música no sistema nervoso central. Cargas submáximas constantes podem permitir que em alguns momentos a música possa competir com as informações fisiológicas advindas das alterações ocasionadas pelo exercício, 
provocando um decréscimo no ritmo de aumento da PSE (Hutchinson \& Tenenbaum, 2007; Tenenbaum et al., 2004). Um atraso na chegada da percepção máxima durante o exercício submáximo de carga constante permitiria o indivíduo prolongar o tempo de exaustão. Entretanto, testes incrementais como o utilizado nesse estudo pode vir a impedir a competição seletiva pelo processamento da informação no sistema nervoso central, devido aos constantes aumentos de carga e desafio imposto aos sistemas corporais. É demonstrado na literatura que a intensidade da carga de exercício é fator importante para a acima referida competição seletiva de informações (Hutchinson \& Tenenbaum, 2007). Nossos dados sustentam esta afirmação.

Tendo em vista o protocolo experimental alvo do presente trabalho, podemos concluir que a utilização da música de gênero "eletrônica" durante um teste incremental até a exaustão não surtiu efeito sobre as variáveis analisadas, apesar dos voluntários relatarem maior motivação nessa situação. Esse tipo de música é comumente utilizado em academias, em que diversas pessoas se exercitam ouvindo a mesma música. Sugere-se maior preocupação com os quatro fatores mencionados em uma recente revisão de Terry e Karageorghis (2006), que podem ser fundamentais na hora se de escolher uma música para a prática esportiva: ritmo, musicalidade, impacto cultural, e associação. Nakamura et al. (2010) mostraram que o gosto musical afeta a presença ou não do efeito ergogênico por ela provocada. Ao se exercitar com músicas de seu agrado, os sujeitos tiveram maior desempenho quando comparado às músicas que eles não gostavam. Utilizamos uma mesma sequência de música para todos, o que pode também ter influenciado os resultados, devido a diferentes gostos pessoais. Futuros estudos com maior cautela nesse ponto, assim como utilização de diferentes protocolos devem ser feitos para elucidar cada vez mais os mecanismos pelos quais a música age no ser humano durante a prática esportiva.

\section{REFERÊNCIAS}

Ashe, M. C., Scroop, G. C., Frisken, P. I., Amery, C. A., Wilkins, M. A., \& Khan, K. M. (2003). Body position affects performance in untrained cyclists. British Journal of Sports Medicine, 37(5), 441-444.

Borg, G. (1970). Perceived exertion as an indicator of somatic stress. Scandinavian Journal of Rehabilitation Medicine, 2(2), 92-98.

Borg, G. (1982). Psychophysical bases of perceived exertion. Medicine $\mathcal{E}$ Science in Sports $\mathcal{E}$ Exercise, 14(5), 377-381.

Boutcher, S. H., \& Trenske, M. (1990). The effects of sensory deprivation and music on perceived exertion and affect during exercise. Journal of Sport and Exercise Psychology, 12 (2), 167-176.

Cacioppo, J. T., Tassinary, L. G., \& Berntson, G. G. (2000). Handbook of psychophysiology ( $3^{\text {rd }}$ ed.). New York: Cambridge University Press.

Cepeda, M. S., Carr, D. B., Lau, J., \& Alvarez, H. (2006). Music for pain relief. Cochrane Database Systematic Reviews, 2. doi: 10.1002/14651858. CD004843.pub2

Copeland, B. L., \& Franks, B. D. (1991). Effects of types and intensities of background music on treadmill endurance. Journal of Sports Medicine and Physical Fitness, 31 (1), 100-103.

Etzel, J. A., Johnsen, E. L., Dickerson, J., Tranel, D., \& Adolphs, R. (2006). Cardiovascular and respiratory responses during musical mood induction. International Journal of Psychophysiology, 61 (1), 57-69.

Garcin, M., Fleury, A., Mille-Hamard, L., \& Billat, V. (2005). Sex-related differences in ratings of perceived exertion and estimated time limit. International Journal of Sports Medicine, 26(8), 675-681.

Hermens, H. J., Freriks, B., Disselhorst-Klug, C., \& Rau, G. (2000). Development of recommendations for SEMG sensors and sensor placement procedures. Journal of Electromyography and Kinesiology, 10(5), 361-374.

Hernandez-Peon, R., Brust-Carmona, H., PenalozaRojas, J., \& Bach-Y-Rita, G. (1961). The efferent control of afferent signals entering the central nervous system. Annals of the New York Academy of Sciences, 89, 866-882.

Hutchinson, J. C., \& Tenenbaum, G. (2007). Attention focus during physical effort: The mediating role of task intensity. Psychology of Sport and Exercise, 8(2), 223-245. 
Karageorghis, C., \& Terry, P. C. (1997). The psychophysical effects of music in sport and exercise. Journal of Sport Behavior, 20(1), 54-68.

Karageorghis, C., Jones, L., \& Low, D. C. (2006). Relationship between exercise heart rate and music tempo preference. Research Quarterly for Exercise and Sport, 77(2), 240-250.

Karageorghis, C., Jones, L., \& Stuart, D. P. (2008). Psychological effects of music tempi during exercise. International Journal of Sports Medicine, 29(7), 613-619.

Kayser, B. (2003). Exercise starts and ends in the brain. European Journal of Applied Physiology, 90(3/4), 411-419.

Khalfa, S., Roy, M., Rainville, P., Dalla Bella, S., \& Peretz, I. (2008). Role of tempo entrainment in psychophysiological differentiation of happy and sad music? International Journal of Psychophysiology, 68(1), 17-26.

Klassen, J. A., Liang, Y., Tjosvold, L., Klassen, T. P., \& Hartling, L. (2008). Music for pain and anxiety in children undergoing medical procedures: A systematic review of randomized controlled trials. Ambulatory Pediatrics, 8(2), 117-128.

Lee, K. P. (1989). The effects of musical tempos on psychophysical responding during sub-maximal treadmill running. Master Thesis, Pennsylvania State University, Pennsylvania, USA.

Lucaccini, L. F., \& Kreit, L. H. (1972). Music. In W. P. Morgan (Ed.), Ergogenic aids and muscular performance (pp. 240-245). New York: Academic.

Lucia, A., Sanchez, O., Carvajal, A., \& Chicharro, J. L. (1999). Analysis of the aerobic-anaerobic transition in elite cyclists during incremental exercise with the use of electromyography. British Journal of Sports Medicine, 33 (3), 178-185.

Mammarella, N., Fairfield, B., \& Cornoldi, C. (2007). Does music enhance cognitive performance in healthy older adults? The Vivaldi effect. Aging Clinical and Experimental Research, 19(5), 394-399.

Nakamura, P. M., Pereira, G., Papini, C. B., Nakamura, F. Y., \& Kokubun, E. (2010). Effects of preferred and nonpreferred music on continuous cycling exercise performance. Perceptual and Motor Skills, 110(1), 257-264.
Noakes, T. D., St Clair Gibson, A., \& Lambert, E. V. (2005). From catastrophe to complexity: A novel model of integrative central neural regulation of effort and fatigue during exercise in humans - Summary and conclusions. British Journal of Sports Medicine, 39(2), 120-124.

Random House (2005). Random House Webster's unabridged dictionary ( $2^{\text {nd }}$ ed.). New York: Autor.

Sarkamo, T., Tervaniemi, M., Laitinen, S., Forsblom, A., Soinila, S., \& Mikkonen, M. (2008). Music listening enhances cognitive recovery and mood after middle cerebral artery stroke. Brain, 131, 866-876.

Simpson, S. D., \& Karageorghis, C. I. (2006). The effects of synchronous music on 400-m sprint performance. Journal of Sports Sciences, 24(10), 1095-1102.

St Clair Gibson, A., \& Noakes, T. D. (2004). Evidence for complex system integration and dynamic neural regulation of skeletal muscle recruitment during exercise in humans. British Journal of Sports Medicine, 38(6), 797-806.

Szmedra, L., \& Bacharach, D. W. (1998). Effect of music on perceived exertion, plasma lactate, norepinephrine and cardiovascular hemodynamics during treadmill running. International Journal of Sports Medicine, 19(1), 32-37.

Tenenbaum, G., Lidor, R., Lavyan, N., Morrow, K., Tonnel, S., \& Gershgoren, A. (2004). The effect of music type on running perseverance and coping with effort sensations. Psychology of Sport and Exercise, 5(2), 89-109.

Terry, P. C., \& Karageorghis, C. I. (2006). Psychophysical effects of music in sport and exercise: An update on theory, research and application. In M. Katsikitis (Ed.), Psychology bridging the Tasman: Science, culture and practice Proceedings of the 2006 Joint Conference of the Australian Psychological Society and the New Zealand Psychological Society (pp. 415-419). Melbourne, VIC: Australian Psychological Society. Yamashita, S., Iwai, K., Akimoto, T., Sugawara, J., \& Kono, I. (2006). Effects of music during exercise on RPE, heart rate and the autonomic nervous system. Journal of Sports Medicine and Physical Fitness, 46(3), 425-430.

(c) EY-No Todo o conteúdo da revista Motricidade está licenciado sob a Creative Commons, excepto quando especificado em contrário e nos conteúdos retirados de outras fontes bibliográficas. 